\title{
Belgeo
}

Revue belge de géographie

\section{Phénomènes d'érosion fluviale et conditions hydrodynamiques de la rivière Saint-Maurice, Québec, Canada}

Fluvial erosion phenomena and hydrodynamic conditions in the Saint-Maurice

river, Quebec, Canada

Diane Saint-Laurent, Michel Bérubé, Isabelle Thériault et Michel Lemieux

\section{OpenEdition}

Journals

Édition électronique

URL : http://journals.openedition.org/belgeo/16018

DOI : 10.4000/belgeo.16018

ISSN : 2294-9135

Éditeur :

National Committee of Geography of Belgium, Société Royale Belge de Géographie

Édition imprimée

Date de publication : 30 juin 2002

Pagination : 109-132

ISSN : $1377-2368$

Référence électronique

Diane Saint-Laurent, Michel Bérubé, Isabelle Thériault et Michel Lemieux, « Phénomènes d'érosion fluviale et conditions hydrodynamiques de la rivière Saint-Maurice, Québec, Canada », Belgeo [En ligne], 2 | 2002, mis en ligne le 01 juillet 2002, consulté le 19 avril 2019. URL : http://journals.openedition.org/ belgeo/16018 ; DOl : 10.4000/belgeo.16018

Ce document a été généré automatiquement le 19 avril 2019

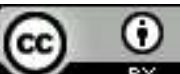

Belgeo est mis à disposition selon les termes de la licence Creative Commons Attribution 4.0 International. 


\section{Phénomènes d'érosion fluviale et conditions hydrodynamiques de la rivière Saint-Maurice, Québec, Canada}

Fluvial erosion phenomena and hydrodynamic conditions in the Saint-Maurice river, Quebec, Canada

Diane Saint-Laurent, Michel Bérubé, Isabelle Thériault et Michel Lemieux

1 Au Québec, les problèmes associés aux phénomènes d'érosion riveraine sont répandus et affectent de nombreux cours d'eau, en particulier ceux qui traversent les dépôts argileux d'origine marine. Les sédiments marins déposés lors de l'épisode de la Mer de Champlain (8000 ans BP) sont particulièrement sensibles à l'érosion riveraine et il n'est pas rare d'observer d'importants mouvements de masse le long des berges constituées de ces argiles marines. La rivière Saint-Maurice dans son cours inférieur circule dans ce type de dépôts et on peut y voir de nombreux glissements ou éboulements le long des pentes riveraines. Dans la partie aval de la rivière Saint-Maurice, entre les rapides Manigance et les rapides des Hêtres, on estime que plus de $65 \%$ des berges sont actives et qu'elles sont affectées à divers degrés par l'érosion riveraine. Cette portion aval de la rivière SaintMaurice est caractérisée par la prédominance des berges argileuses ou argilo-limoneuses très hautes $(8-10 \mathrm{~m})$ et fortement inclinées $\left(>25^{\circ}\right)$. La composition des matériaux et la géométrie des talus riverains expliquent en grande partie la vulnérabilité des berges à l'érosion riveraine dans ce secteur.

2 Afin de mieux comprendre la dynamique fluviale et les facteurs en cause dans l'explication des phénomènes de l'érosion riveraine, une étude détaillée sur les caractéristiques morpho-sédimentologiques des berges et les conditions hydrodynamiques de la rivière a été réalisée. On examine l'influence des vagues et des courants et l'action des glaces et des variations du niveau d'eau de la rivière sur l'érosion des berges dans les conditions actuelles. D'autres études sur l'érosion des berges pourront 
éventuellement être réalisées lors de la mise en service de la nouvelle centrale GrandMère prévue en 2004.

\section{Localisation et contexte géomorphologique}

La zone d'étude couvre le tronçon sud de la rivière Saint-Maurice situé entre les rapides Manigance et les rapides des Hêtres (figure 1). Ces derniers rapides se situent en aval de la centrale hydroélectrique de Grand-Mère ( $\mathrm{km} \mathrm{49)}$, à quelques $8 \mathrm{~km}$ au sud, alors que les rapides Manigance $(\mathrm{km} 88)$ délimitent le bief amont situé à $39 \mathrm{~km}$ environ au nord de la centrale. Soulignons que la rivière Saint-Maurice est largement régularisée avec à sa tête le réservoir Gouin et sept centrales distribuées le long de son parcours. L'ensemble de la rivière couvre $587 \mathrm{~km}$ de longueur, traversant dans sa partie nord les contreforts des Laurentides pour rejoindre la plaine argileuse des Basses-Terres du Saint-Laurent. La portion nord du plateau Laurentien est caractérisée par un relief de vallons et de collines qui s'estompe progressivement vers la plaine du Saint-Laurent.

Figure 1. Carte de localisation de la zone d'étude avec les segments riverains étudiés.

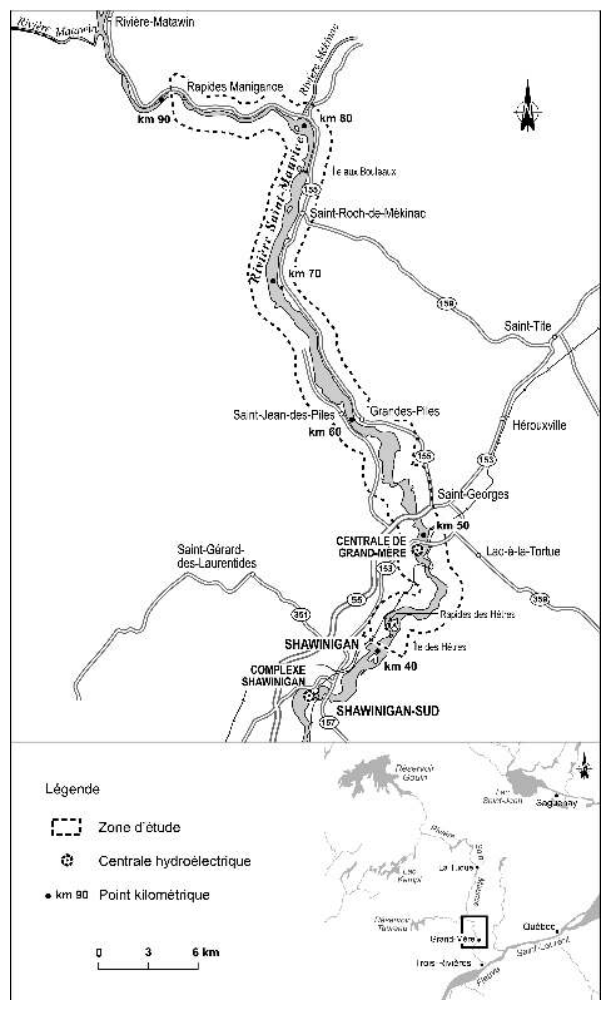

Les dépôts quaternaires qui recouvrent le socle rocheux ont été principalement mis en place lors de la glaciation wisconsinienne ( $\sim 2500$ ans BP) et durant la transgression marine (Mer de Champlain) qui a suivi cette période glaciaire (Vincent, 1989). Les eaux de la Mer de Champlain ont envahi la haute vallée du Saint-Laurent pénétrant la vallée du Saint-Maurice jusque dans la région de La Tuque. Durant la transgression marine, des sédiments fins d'eau profonde (argile et limon) de plusieurs mètres d'épaisseur (entre 8 et 15 mètres) ont recouvert le socle et les dépôts d'origine glaciaire, fluvioglaciaire ou juxtaglaciaire. Lors du retrait des eaux de la Mer de Champlain, des sables deltaïques ou pro-deltaïques (ancien complexe deltaïque à l'embouchure de la rivière Saint-Maurice) se 
sont accumulés et ont recouvert les sédiments marins. Le relèvement isostatique a permis à la rivière d'entailler ces dépôts meubles jusqu'au niveau actuel (Vincent, 1989). Les relevés stratigraphiques effectués dans le couloir de la rivière Saint-Maurice, près du pont de Grand-Mère, révèlent une certaine régularité dans les séquences sédimentaires (H-Q, 1995). Les dépôts marins à dominance silto-argileuse reposent sur le socle rocheux ou sur des dépôts glaciaires ou fluvioglaciaires, surmontés de silts pro-deltaïques et des sables deltaïques. Les levés géotechniques effectués en rive droite près de Grand-Mère s'apparentent à cette séquence sédimentaire, soit des dépôts silto-argileux d'origine marine ( $8 \mathrm{~m}$ d'épaisseur) sous des dépôts sableux $(9 \mathrm{~m})$ de la terrasse supérieure (ibid., 1995).

\section{Conditions hydrologiques et hydrodynamiques}

\section{Variations du niveau de la rivière}

La rivière Saint-Maurice est soumise à une gestion contrôlée par les différents biefs localisés le long du tronçon entre La Tuque et l'embouchure de la rivière qui se jette dans le fleuve Saint-Laurent (figure 1). Dans le secteur d'étude, le bief amont de la centrale de Grand-Mère forme une réserve utile de $27 \mathrm{hm}^{3}$ utilisée aux fins des besoins hydroélectriques. Ce réservoir est qualifié d'aménagement de type « au fil de l'eau » et le laminage des crues se fait principalement par le barrage Gouin, en tête du bassin. Le niveau d'eau dans le bief amont demeure relativement constant (tableau 1) avec des niveaux minimal et maximal qui varient entre $99,52 \mathrm{~m}$ et 103,27 $\mathrm{m}$ respectivement, ce qui représente un marnage de 3,75 m. Durant la période hivernale, les niveaux du réservoir sont maintenus près de la cote maximale. Dans le bief aval de la centrale, le niveau d'eau est contrôlé par le débit en provenance de la centrale et de l'évacuateur (turbiné et déversé) et par le niveau du seuil et la largeur de la rivière à l'endroit des rapides des Hêtres. Les niveaux moyens mensuels varient relativement peu d'un mois à l'autre (tableau 1), et ce sont surtout durant les mois d'avril et de mai que les niveaux atteignent leur maximum ( $\pm 78,10$ et $\pm 78,72 \mathrm{~m}$ ), pour ensuite atteindre le niveau minimal durant les mois d'août et septembre $( \pm 77,42$ et $\pm 77,38 \mathrm{~m})$. 
Tableau 1. Niveaux mensuels minimum, moyen et maximum pour les biefs amont et aval de la centrale de Grand-Mère.

\begin{tabular}{|c|c|c|c|c|c|c|}
\hline \multirow[t]{2}{*}{ MOIS } & \multicolumn{3}{|c|}{$\begin{array}{l}\text { NIVEAU BIEF AMONT } \\
(\mathrm{m})\end{array}$} & \multicolumn{3}{|c|}{$\begin{array}{l}\text { NIVEAU BIEF AVAL } \\
(\mathrm{m})\end{array}$} \\
\hline & Minimum & Moyen & Maximum & Minimum & Moyen & Maximum \\
\hline Janvier & 100,39 & 102,13 & 103,27 & 75,74 & 77,69 & 78,44 \\
\hline Février & 100,55 & 102,12 & 102,57 & 75,80 & 77,72 & 79,07 \\
\hline Mars & 100,64 & 102,14 & 102,54 & 75,71 & 77,65 & 79,13 \\
\hline Avril & 100,22 & 102,14 & 102,77 & 68,70 & 78,10 & 82,66 \\
\hline Mai & 100,46 & 102,21 & 102,93 & 76,23 & 78,72 & 83,53 \\
\hline Juin & 100,43 & 102,18 & 102,57 & 75,86 & 77,79 & 81,81 \\
\hline Juillet & 99,83 & 102,09 & 102,60 & 75,38 & 77,47 & 81,11 \\
\hline Août & 100,24 & 102,10 & 102,57 & 75,65 & 77,42 & 79,17 \\
\hline Septembre & 99,52 & 102,10 & 103,08 & 74,83 & 77,38 & 78,70 \\
\hline Octobre & 100,37 & 102,13 & 102,55 & 75,53 & 77,51 & 81,32 \\
\hline Novembre & 100,46 & 102,16 & 102,59 & 75,38 & 77,58 & 80,10 \\
\hline Décembre & 100,11 & 102,09 & 102,57 & 74,41 & 77,63 & 79,52 \\
\hline
\end{tabular}

\section{Caractéristiques du couvert de glace}

6 Les études effectuées lors des survols hivernaux sur la rivière Saint-Maurice dans le secteur d'étude (Groupe-Conseil LaSalle Inc., 1999 ; H-Q, 1999, 1996abc, 1993b ; Guerrero, 1995) indiquent que le couvert de glace se forme assez rapidement dans le bief amont, depuis la centrale jusqu'à la confluence de la rivière Mékinac, soit vers la mi-décembre. De la rivière Mékinac aux rapides Manigance, le couvert de glace se forme plus lentement, en raison de la vitesse des courants de plus en plus élevée vers l'amont. Le couvert de glace progresse vers l'amont, alimenté par les glaces qui dérivent depuis La Tuque ( $\mathrm{H}-\mathrm{Q}$, 1999, p. 5/22). En général, le bief amont se couvre de glaces entièrement à la mi-janvier, sauf dans les zones des rapides. Dans le bief aval, le couvert de glace se forme vers la midécembre, entre les rapides des Hêtres et Shawinigan, et plus tardivement, en aval de la centrale de Grand-Mère. Dans le secteur immédiatement en aval de la centrale, la couverture de glace progresse vers le centre entre les mois de janvier et mars. Cependant une zone d'eau libre persiste en aval de la centrale sur environ 100 à $300 \mathrm{~m}$. Plus en aval, certaines sections de la rivière demeurent également libres de glace, notamment dans le bras central des rapides des Hêtres.

\section{Méthodes et approches}

7 Les divers rapports techniques réalisés sur l'étude des berges et les conditions hydrauliques du Saint-Maurice ont été consultés pour réaliser une synthèse préliminaire (H-Q, 1999 ; Groupe-Conseil LaSalle Inc., 1999 ; H-Q, 1996abc, 1995, 1993ab ; Guerrero, 1995 ; Tecsult inc., 1996 ; Groupe HBA, 1993 ; Société d'Ingénierie Shawinigan Ltée, 1974). Les données les plus pertinentes contenues dans ces rapports ont été intégrées à l'étude des berges afin de regrouper toutes les informations utiles à cette analyse. Ces données concernent plus spécifiquement les coupes topographiques effectuées le long des rives en amont et en aval de la centrale, les analyses granulométriques, les données sur le régime des glaces et les études hydrologiques et hydrauliques réalisées dans le secteur d'étude. 


\section{Le calcul de vagues et l'érosion des berges}

Les données anémométriques de la station météorologique(1) de Trois-Rivières (Environnement Canada, 1982) ont été utilisées pour élaborer les modèles mathématiques servant au calcul de la force érosive des vagues sur les berges. Les données anémométriques retenues (vitesse et direction du vent de 1963-1977 et de 1991 à 1996) ont été transformées en données de vents horaires, afin de tenir compte de la durée nécessaire à la formation des vagues. La répartition statistique des vents maximum annuels $(\mathrm{km} / \mathrm{h})$ a été effectuée pour chacune des huit directions de la rose des vents, à partir des valeurs maximales annuelles enregistrées pour chaque direction, suivant la loi statistique de Gumbel (figures 2a et 2b). Cette loi peut s'appliquer pour des séries courtes de données, bien qu'il soit préférable d'utiliser des séries longues (50 ans ou plus) pour des récurrences centennales, bien qu'il demeure difficile d'obtenir des séries longues pour les données anémométriques. Enfin, les données utilisées sont valables pour la partie sud de la zone d'étude, c'est-à-dire des rapides des Hêtres jusqu'à Grandes-Piles. Plus au nord, une attention doit être apportée à l'effet de couloir créé par l'encaissement de la vallée, qui canalise les vents et peut faire ainsi augmenter leur vitesse.

9 Les calculs pour évaluer la longueur de la partie submergée de la berge soumise à l'érosion des vagues suivent les principes suivants :

10 En premier lieu, les hauteurs, les longueurs d'onde et les périodes des vagues sont déterminées à partir des vents d'une durée d'une heure, en supposant que la hauteur des vagues générées n'est pas influencée par la profondeur d'eau. En second lieu, on vérifie si la profondeur d'eau influence la croissance des vagues et si la durée d'une heure est suffisante pour que les vagues soient pleinement développées. Les caractéristiques des vagues ont donc été évaluées pour chacune des directions pour lesquelles les vents ont une emprise.

11 La hauteur significative des vagues Hs, qui représente la moyenne d'un tiers des plus hautes vagues, est donnée par la relation suivante :

$$
\frac{g H S}{U_{A}^{2}}=\alpha\left(\frac{g F}{U_{A}^{2}}\right)^{\beta}
$$

13 où :

$U_{A}=$ la vitesse de friction du vent sur l'eau (en $\left.\mathrm{m} / \mathrm{s}\right)$;

$F=$ le fetch effectif (en mètres) ;

$G=$ l'accélération gravitationnelle;

$H s=$ la hauteur significative des vagues.

14 Des études réalisées par la Société d'Énergie de la Baie James (SEBJ, 1975) ont permis de valider les coefficients $\alpha$ et $\beta$ pour les réservoirs du nord du Québec. Cette étude propose les coefficients suivants :

$15 \alpha=3,1 \times 10-3$

$\beta=0,4$ 
16 Par ailleurs, cette étude recommande d'utiliser un fetch effectif correspondant à la moyenne arithmétique de la longueur du fetch de 41 radiales espacées de 1 degré ; la radiale principale correspondant à la direction du vent.

La période de la vague $T$ est calculée à partir de la relation :

$$
\frac{g T}{U_{A}}=0,2857 \frac{g F^{1 / 3}}{U_{A}}
$$

$18 \quad(2)$

19 La longueur d'onde $L$ est donnée par la relation :

$$
d_{1}=\frac{H}{\sinh \left(\frac{2 \pi d}{L}\right)}
$$

20 (3)

où $d$ = la profondeur d'eau;

tanh : tangente hyperbolique

21 À l'approche du rivage, la hauteur et la longueur d'onde de la vague subissent des transformations dues à l'influence de la profondeur d'eau. L'influence du frottement et le changement de direction du front d'onde causé par les contours bathymétriques ont été négligés, étant donné la faible longueur de la zone dite peu profonde. La hauteur de la vague après transformation devient :

$$
H=H_{0} \sqrt{\frac{1}{0,5\left(\frac{1+\frac{4 \pi d}{L}}{\sinh \left(\frac{4 \pi d}{L}\right)}\right)}}
$$

22 (4)

où : $\mathrm{H}_{0}$ est la hauteur de la vague générée en eau profonde.

Il est à noter que pour toutes les directions et pour toutes les positions étudiées, la cambrure de la vague (rapport entre sa hauteur et sa longueur) est telle que les vagues ne déferlent pas avant d'atteindre le rivage.

Le mouvement orbital des particules d'eau d'une vague est responsable du déplacement des sédiments. Pour les particules sans cohésion, de diamètre inférieur à $0,5 \mathrm{~mm}$, la relation empirique définie ci-après permet de déterminer la vitesse du mouvement orbital à partir de laquelle se produit l'initiation du mouvement des sédiments (Komar et Miller, 1973) :

$$
\frac{\rho u_{t}^{2}}{\left(\rho_{s}-\rho\right) g D}=0,2 I\left(\frac{d_{e}}{D}\right)^{\prime 2}
$$

où :

$u t=$ la vitesse sur le fond;

$p=$ la densité de l'eau;

$p s$ = la densité des particules constituant le fond ;

$D$ = le diamètre des particules ; 
$g$ = l'accélération gravitationnelle;

do = le diamètre du mouvement orbital de l'eau.

27 Ce dernier est donné par la relation suivante :

$d_{10}=\frac{H}{\sinh \left(\frac{2 \pi d}{l}\right)}$

$28 \quad(6)$

29 À partir de la granulométrie des particules constituant la charge de fond (silt et argile), il devient donc possible, pour des conditions de vagues données, de prédire l'étendue de la partie submergée de la berge qui sera soumise à l'érosion. Les analyses granulométriques réalisées sur les berges indiquent que les matériaux sont constitués surtout de silt et d'argile (Hydro-Québec, 1995). Les calculs de la zone soumise à l'érosion ont donc été faits en utilisant un diamètre des particules de 0,01 $\mathrm{mm}$ et une densité correspondant à 2,7 fois la densité de l'eau. Le tableau 2a indique les longueurs perpendiculaires à partir de la rive et les profondeurs d'eau maximales de la partie submergée de la berge pouvant être affectées par l'érosion, pour chacune des directions offrant une emprise au vent. Il est à noter que ces valeurs ne sont valables que pour des vents de forte intensité, survenant en moyenne une heure par année pour chacune des directions. Le tableau $2 \mathrm{~b}$ montre les longueurs affectées lorsque des vents d'intensité moyenne surviennent. Ces résultats indiquent que les particules fines sont transportées lorsque des vents de forte intensité (récurrence supérieure à deux ans) se produisent, mais que les vents d'intensité moyenne n'induisent pas de phénomènes d'érosion significatifs sur les plages. Le taux de recul de la plage peut difficilement être déduit à partir de ces calculs. Enfin le tableau $2 \mathrm{c}$ fournit un certain nombre de valeurs sur la largeur et la profondeur du chenal pour la zone d'étude (Hydro-Québec, 1995, 1999).

Tableau 2a. Longueur de la partie submergée de la berge soumise à l'érosion suivant le vent maximal annuel.

\begin{tabular}{|c|c|c|c|c|}
\hline \multirow[t]{2}{*}{ DIRECTION } & \multicolumn{2}{|l|}{$\begin{array}{l}\text { KM } 50,5 \\
\text { (rive gauche) }\end{array}$} & \multicolumn{2}{|l|}{$\begin{array}{l}\mathrm{KM} 69,9 \\
\text { (rive droite) }\end{array}$} \\
\hline & $\begin{array}{l}\text { Profondeur } \\
\text { maximale soumise } \\
\text { à l'érosion }(\mathrm{m})\end{array}$ & $\begin{array}{l}\text { Longueur } \\
\text { (m) }\end{array}$ & $\begin{array}{l}\text { Profondeur } \\
\text { maximale soumise } \\
\text { à l'érosion }(\mathrm{m})\end{array}$ & $\begin{array}{l}\text { Longueur } \\
\text { (m) }\end{array}$ \\
\hline Nord & - & - & 6,0 & 60 \\
\hline Nord-est & - & - & 5,5 & 55 \\
\hline Est & - & - & 4,5 & 45 \\
\hline Sud-est & - & - & 2,5 & 25 \\
\hline Sud & 2,6 & 8 & - & . \\
\hline Sud-ouest & 2,9 & 9 & - & . \\
\hline Ouest & 1,9 & 6 & - & . \\
\hline Nord-ouest & 2,4 & 7 & - & - \\
\hline
\end{tabular}


Tableau $2 \mathrm{~b}$. Longueur de la partie submergée de la berge soumise à l'érosion suivant le vent moyen.

\begin{tabular}{|c|c|c|c|c|}
\hline \multirow[t]{2}{*}{ DIRECTION } & \multirow{2}{*}{$\begin{array}{l}\text { KM 50,5 } \\
\text { (rive gauche) }\end{array}$} & $\begin{array}{l}\text { KM 69,9 } \\
\text { (rive droite) }\end{array}$ & & \multirow[b]{2}{*}{$\begin{array}{l}\text { Longueur } \\
\text { (m) }\end{array}$} \\
\hline & & $\begin{array}{l}\text { Longueur } \\
\text { (m) }\end{array}$ & $\begin{array}{l}\text { Profondeur } \\
\text { maximale soumise } \\
\text { à l'érosion (m) }\end{array}$ & \\
\hline Nord & . & . & 0,4 & 4 \\
\hline Nord-est & - & . & 0,4 & 4 \\
\hline Est & - & - & 0,3 & 3 \\
\hline Sud-est & - & - & 0,4 & 4 \\
\hline Sud & - & - & $\therefore$ & - \\
\hline Sud-ouest & - & - & - & - \\
\hline Ouest & 0,4 & 1 & . & . \\
\hline Nord-ouest & 0,2 & 1 & - & . \\
\hline
\end{tabular}

Tableau 2c. Données sur la profondeur et la largeur du chenal dans le secteur d'étude.

\begin{tabular}{|c|c|c|c|}
\hline Localisation & $\begin{array}{l}\text { Profondeur } \\
\text { (m) }\end{array}$ & $\begin{array}{l}\text { Largeur } \\
(\mathrm{m})\end{array}$ & $\begin{array}{l}\text { Nature du fond } \\
\text { (entre } 0.2 \mathrm{~m} \text { ) }\end{array}$ \\
\hline $\begin{array}{l}\text { Pointe aux Ormes } \\
(\mathrm{km} \mathrm{48,0)}\end{array}$ & $\pm 60-75$ & \pm 150 & Roche en place \\
\hline $\begin{array}{l}\text { Aval de la centrale } \\
(\mathrm{km} 48,9)\end{array}$ & \pm 50.75 & \pm 500 & Roche en place \\
\hline $\begin{array}{l}\text { Amont de la centrale } \\
(\mathrm{km} 49,6)\end{array}$ & $\pm 80-100$ & \pm 400 & Sable moyen à galet \\
\hline $\begin{array}{l}\text { Pointe à Desaulniers } \\
(\mathrm{km} 50,5)\end{array}$ & $\pm 90-95$ & \pm 350 & Argile et/ou limon \\
\hline $\begin{array}{l}\text { Baie-à-la-Pêche } \\
(\mathrm{km} 69,9)\end{array}$ & - & \pm 800 & Argile et/ou limon \\
\hline
\end{tabular}

\section{Caractérisation des berges}

La caractérisation des berges a été effectuée à partir des photographies aériennes à l'échelle 1: 15000 (années 1989 et 1993) et des relevés de terrain. Les cartes topographiques à l'échelle du 1:10000 et 1:50 000, et les coupes stratigraphiques ont servi à compléter l'information. La rivière a été subdivisée en segments riverains de façon à représenter le type de dépôts meubles et les caractéristiques géométriques des berges. Les limites des segments riverains déterminent soit un changement dans la nature des matériaux ou dans la géométrie (hauteur et pente) des berges, ou les deux à la fois. La hauteur des talus et le degré des pentes ont été regroupés en fonction de classes distinctes. 


\section{Taux de recul des berges}

31 La comparaison des photographies aériennes prises entre 1964 et 1987 ou 1993 pour différents segments riverains montrant les berges les plus affectées par l'érosion fluviale, a permis d'évaluer le taux de recul des berges depuis une trentaine d'années. Cette évaluation s'est faite par la superposition numérique des photos aériennes et par l'ajustement des échelles photographiques à partir des cartes de base à l'échelle $1: 10000$. Cette technique comprend plusieurs étapes, soit :1) la délimitation des lignes de rivage sur chacune des photos aériennes; 2) l'identification des points de référence sur l'ensemble des photos ; 3) la numérisation par balayage ; 4) la correction géométrique des photos par superposition des points de référence; et 5) la numérisation à l'écran de l'ordinateur des lignes de rivage. La figure 3 montre un exemple de numérisation des photographies aériennes pour le segment situé entre le km 64,0 et 67,0. Soulignons que la numérisation des photographies à l'échelle du $1: 15000$ est soumise à une certaine marge d'erreur étant donné la délimitation des lignes de rivage à cette échelle photographique.

Figure 3. Exemple d'une photographie aérienne numérisée $(\mathrm{km} 60,0)$ montrant le taux de recul des berges.

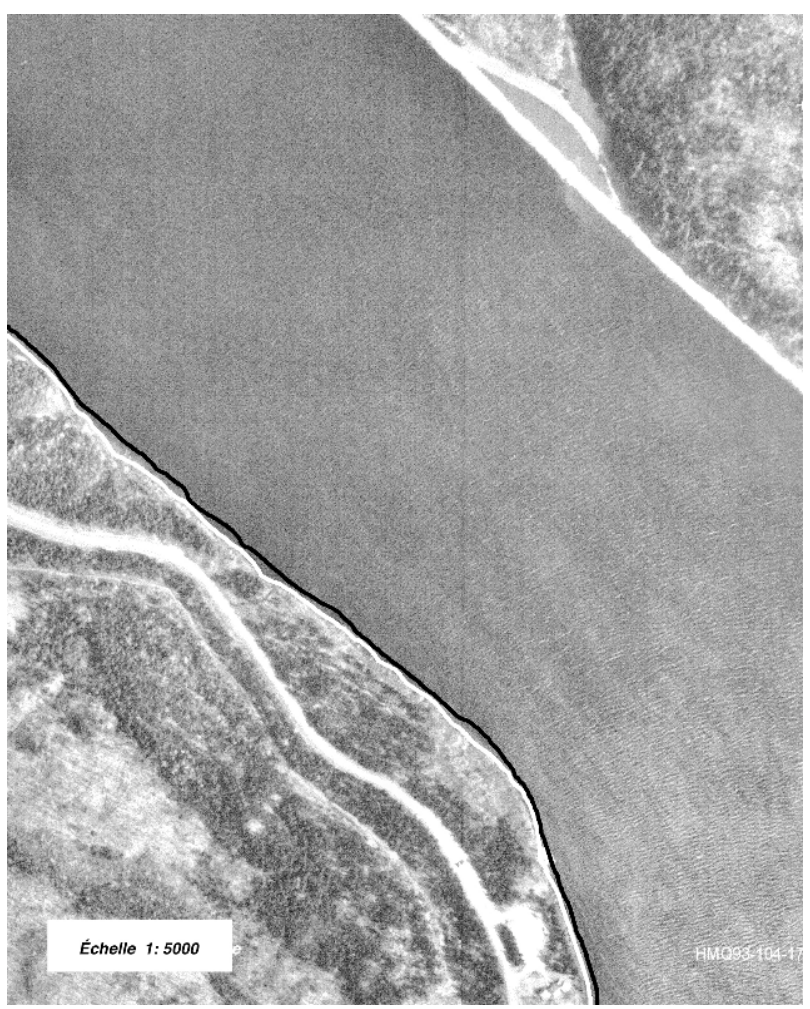

\section{Classes d'érosion des berges}

Les observations faites sur le terrain ont permis de regrouper les berges en quatre classes qui correspondent à divers degrés d'érosion (négligeable, faible, moyenne et forte). Les indices visuels ont servi de facteur discriminant dans ce classement des berges soumis à l'érosion puisqu'ils reflètent les conditions actuelles, tandis que le taux de recul reflète 
davantage les conditions passées et actuelles. Les indices visuels s'appuient essentiellement sur la gravité des phénomènes observés. Ainsi, par exemple, une berge affectée par un glissement ou un éboulement a été classée parmi les «berges à forte érosion "; tandis qu'une berge soumise à un faible sapement a été classée parmi les «berges à faible érosion »; une berge montrant un sapement important à la base mais dont la pente est faiblement inclinée a été classée parmi les « berges à érosion modérée »; enfin, une berge ayant une large plage et peu soumise à l'action des vagues (baie protégée des vents ou par la végétation par exemple) a été classée parmi les «berges à érosion négligeable ». Le tableau 3 fournit les critères visuels utilisés pour mieux définir les divers degrés d'érosion des berges observés sur le terrain. Ce regroupement s'appuie sur une appréciation qualitative du degré d'érosion des berges et non pas sur des valeurs quantifiées, tel que le volume métrique de matériaux érodés par exemple (Saint-Laurent et Guimont, 1999).

Tableau 3. Critères visuels utilisés pour définir les degrés d'érosion des berges.

\begin{tabular}{|c|c|c|}
\hline $\begin{array}{l}\text { Degrés d'érosion } \\
\text { des berges }\end{array}$ & Critères visuels & Autres caractéristiques \\
\hline Négligeable & Sapement faible à la base du talus & $\begin{array}{l}\text { Présence d'une plage et/ou } \\
\text { de plantes aquatiques ou } \\
\text { semi-aquatiques protégeant } \\
\text { la berge; rive protégée des } \\
\text { vagues et courants }\end{array}$ \\
\hline Faible & Sapement à la base du talus & $\begin{array}{l}\text { Arbres légèrement inclinés, } \\
\text { présence fréquente d'une plage } \\
\text { et/ou d'une végétation riveraine }\end{array}$ \\
\hline Modéré & $\begin{array}{l}\text { Sapement à la base du talus et } \\
\text { éboulements superficiels le long } \\
\text { de la pente }\end{array}$ & $\begin{array}{l}\text { Arbres inclinés, pentes } \\
\text { partiellement dénudées } \\
\text { de végétation; pente } \\
\text { modéré à forte (cf. figure } 4 \mathrm{a} \text { ) }\end{array}$ \\
\hline Fort & $\begin{array}{l}\text { Éboulements et/ou glissements } \\
\text { le long du talus; } \\
\text { présence fréquente de bourrelets } \\
\text { de matériaux au pied du talus }\end{array}$ & $\begin{array}{l}\text { Arbres déracinées et/ou } \\
\text { fortement inclinés; } \\
\text { pentes généralement dénudées } \\
\text { de couverture végétale; } \\
\text { pente généralement forte } \\
\left(>25^{\circ}\right) \text { (cf. figure } 4 \mathrm{~b} \text { ) }\end{array}$ \\
\hline
\end{tabular}

\section{Résultats et discussions}

\section{Caractéristiques morpho-sédimentologiques des berges}

La caractérisation des berges du secteur d'étude a été réalisée en tenant compte de la nature des matériaux qui composent les berges et leur géométrie (tableaux $4 \mathrm{a}$ et 4b). Les données compilées regroupent autant les berges naturelles que les berges artificielles, dont les proportions sont de $80 \%$ et $20 \%$ respectivement. La compilation des données s'est faite en tenant compte également de la répartition amont et aval des berges en fonction de la centrale de Grand-Mère, étant donné que les conditions hydrologiques diffèrent de part et d'autre de la centrale. Les matériaux des berges naturelles sont principalement constitués de silt argileux, d'argile silteuse ou de sable (tableaux 4a et 4b). 
On trouve également du roc sur plus de $23 \mathrm{~km}$ de longueur, localisé à divers endroits le long de la rivière, notamment aux abords de la centrale (H-Q, 1995). Les berges constituées de silt argileux ou d'argile silteuse dominent tout le bief amont de la centrale, sur la rive droite en particulier, soit 20,7 km comparativement à 9,0 km sur la rive gauche (tableau 4a). De l'île aux Bouleux jusqu'aux rapides Manigance, les berges sont composées surtout de sable ou de sable graveleux. Elles sont deux fois plus nombreuses en rive gauche ( $8,3 \mathrm{~km}$ de longueur) qu'en rive droite $(4,1 \mathrm{~km})$. Dans le bief aval de la centrale, ce sont les berges sableuses qui dominent ; elles représentent $46 \%$ des berges de ce tronçon. Enfin, les berges artificielles se retrouvent sur près de $27 \mathrm{~km}$ de longueur pour l'ensemble du tronçon et elles sont constituées d'enrochement et de matériaux divers (tableaux 4a et ab). Le nombre élevé de berges formées d'enrochement en amont de la centrale s'explique par l'aménagement de la route 155 qui longe la rive gauche de la rivière Saint-Maurice sur plus de $12 \mathrm{~km}$ de longueur.

Afin de dresser un portrait plus précis des berges soumises à l'érosion, un regroupement des berges en classe d'érosion a été réalisé en tenant compte de la hauteur des talus et de l'inclinaison des pentes et de leur localisation en amont et en aval de la centrale (tableaux 5 et 6). Les berges naturelles caractérisées par des talus bas ou moyennement élevés, soit de 0 à $<2 \mathrm{~m}$ et de 2 à $10 \mathrm{~m}$ de hauteur représentent plus de $75 \%$ des berges (tableau 5). En amont de la centrale, elles couvrent 56,1 km de longueur et en aval 10,1 km. Les berges avec des hauts talus $(>10 \mathrm{~m})$ occupent presque exclusivement le bief amont de la centrale et on les retrouve surtout en rive droite. Le bief amont de la centrale est caractérisé par un plus grand nombre de berges en pente forte $\left(>25^{\circ}\right)$. Ces berges occupent $46,0 \mathrm{~km}$ de longueur de rive dans le bief amont et 5,8 $\mathrm{km}$ en aval (tableau 6), représentant ensemble plus de $60 \%$ de berges constituées de matériaux argileux ou sableux. Ces types de berges sont particulièrement sensibles aux mouvements de masse, surtout lorsque les talus sont fortement inclinés $\left(>25^{\circ}\right)$.

\section{Classification des berges affectées par l'érosion}

35 À partir des relevés de terrain et de la numérisation des données sur les feuillets cartographiques, il a été possible de localiser et de dénombrer les zones actives d'érosion des berges et les zones stables ou protégées. Les berges affectées par l'érosion riveraine représentent plus de $62 \%$ des berges de la zone d'étude, soit $85,2 \mathrm{~km}$ sur $135,6 \mathrm{~km}$ de longueur. Elles se retrouvent principalement en amont de la centrale de Grand-Mère et en rive droite (tableau 7). Les berges sont affectées principalement par trois phénomènes, soit: (1) le sapement des vagues au pied des talus qui cause, habituellement, le déchaussement des arbres riverains (figure 4a); (2) les éboulements le long des pentes abruptes (figure $4 \mathrm{~b}$ ) ; (3) et les glissements de terrain. On peut d'ailleurs observer les cicatrices d'anciens glissements dans la partie nord de la zone d'étude.

Dans le bief amont, les berges soumises à une forte érosion représentent $8,9 \mathrm{~km}$ de longueur sur un total de $74,7 \mathrm{~km}$, incluant les îles, tandis que dans le bief aval, les berges sont assez peu affectées par l'érosion (tableau 7). Si l'on compare les berges du bief amont à celles du bief aval, on constate rapidement que les berges du bief amont (surtout constituées de matériaux argileux ou sableux, et avec des pentes fortement inclinées) sont plus affectées par les phénomènes d'érosion. En effet, les terrasses d'argile ou celles constituées de sable sont en grand nombre affectées par des éboulements ou des décrochements le long des pentes. Dans le bief aval, la nature des berges (matériaux 
glaciaires, affleurements rocheux, etc.) fait en sorte que les berges sont moins vulnérables à l'érosion. Par ailleurs, dans le bief aval, le lit de la rivière a atteint son niveau de base, ce qui explique l'abondance des affleurements rocheux à cet endroit.

Tableau 4a. Longueur des berges naturelles et artificielles suivant les classes de matériaux (section amont).

\begin{tabular}{|c|c|c|c|c|c|c|c|c|}
\hline \multicolumn{9}{|c|}{ AMONT } \\
\hline \multirow[t]{2}{*}{ MATÉRIAUX } & \multicolumn{3}{|c|}{ LONGUEUR DES BERGES (km) } & \multicolumn{5}{|c|}{ POURCENTAGE DE LA LONGUEUR TOTALE } \\
\hline & $\begin{array}{c}\text { Rive } \\
\text { gauche }\end{array}$ & $\begin{array}{c}\text { Rive } \\
\text { droite }\end{array}$ & Iles & Total & $\begin{array}{c}\text { Rive } \\
\text { gauche }\end{array}$ & $\begin{array}{l}\text { Rive } \\
\text { droite }\end{array}$ & Iles & total \\
\hline Berge naturelle & & & & & & & & \\
\hline $\begin{array}{l}\text { Silt argileux ou argile } \\
\text { silteuse (CM) }\end{array}$ & 0,3 & 0,8 & 0,0 & 1,1 & 0,3 & 0,7 & 0,0 & 1,0 \\
\hline $\begin{array}{l}\text { Sable sur silt argileux } \\
\text { (S/CM) }\end{array}$ & 8,7 & 19,9 & 0,0 & 28,6 & 7,5 & 17,5 & 0,0 & 25,0 \\
\hline Sable (S) & 8,3 & 4,1 & 15,6 & 28,0 & 7,1 & 3,7 & 13,7 & 24,5 \\
\hline Sable et gravier (SG) & 8,1 & 8,9 & 0,0 & 17,0 & 7,1 & 7,9 & 0,0 & 15,0 \\
\hline Blocs et cailloux (BCa) & 0,0 & 0,0 & 0,0 & 0,0 & 0,0 & 0,0 & 0,0 & 0,0 \\
\hline $\operatorname{Roc}(\mathrm{R})$ & 7,8 & 7,0 & 0,2 & 15,0 & 6,8 & 6,1 & 0,1 & 13,0 \\
\hline Total & 33,2 & 40,7 & 15,8 & 89,7 & 28,8 & 35,9 & 13,8 & 78,5 \\
\hline Berge artificielle & & & & & & & & \\
\hline Matériaux divers & 0,5 & 3,4 & 0,0 & 3,9 & 0,4 & 3,0 & 0,0 & 3,4 \\
\hline Enrochement & 16,4 & 4,4 & 0,0 & 20,8 & 14,3 & 3,8 & 0,0 & 18,1 \\
\hline Total & 16,9 & 7,8 & 0,0 & 24,7 & 14,7 & 6,8 & 0,0 & 21,5 \\
\hline TOTAL & 50,1 & 48,5 & 15,8 & 114,4 & 43,5 & 42,7 & 13,8 & 100,0 \\
\hline
\end{tabular}

Tableau 4b. Longueur des berges naturelles et artificielles suivant les classes de matériaux (section aval).

\begin{tabular}{|c|c|c|c|c|c|c|c|c|}
\hline \multicolumn{9}{|c|}{ AVAL } \\
\hline \multirow[t]{2}{*}{ MATÉRIAUX } & \multicolumn{3}{|c|}{ LONGUEUR DES BERGES $(\mathrm{km})$} & \multicolumn{5}{|c|}{ POURCENTAGE DE LA LONGUEUR TOTALE } \\
\hline & $\begin{array}{l}\text { Rive } \\
\text { gauche }\end{array}$ & $\begin{array}{l}\text { Rive } \\
\text { droite }\end{array}$ & Iles & Total & $\begin{array}{l}\text { Rive } \\
\text { gauche }\end{array}$ & $\begin{array}{l}\text { Rive } \\
\text { droite }\end{array}$ & lles & total \\
\hline Berge naturelle & & & & & & & & \\
\hline $\begin{array}{l}\text { Silt argileux ou argile } \\
\text { silteuse (CM) }\end{array}$ & 0,0 & 0,0 & 0,0 & 0,0 & 0,0 & 0,0 & 0,0 & 0,0 \\
\hline $\begin{array}{l}\text { Sable sur silt argileux } \\
\text { (S/CM) }\end{array}$ & 0,0 & 0,0 & 0,0 & 0,0 & 0,0 & 0,0 & 0,0 & 0,0 \\
\hline Sable (S) & 5,7 & 3,6 & 0,5 & 9,8 & 26,7 & 17,4 & 2,4 & 46,5 \\
\hline Sable et gravier (SG) & 0,0 & 0,1 & 0,0 & 0,1 & 0,0 & 0,5 & 0,0 & 0,5 \\
\hline Blocs et cailloux (BCa) & 0,0 & 0,0 & 0,6 & 0,6 & 0,0 & 0,0 & 2,8 & 2,8 \\
\hline $\operatorname{Roc}(\mathrm{R})$ & 3,0 & 3,0 & 2,5 & 8,5 & 14,1 & 14,1 & 11,7 & 39,9 \\
\hline Total & 8,7 & 6,7 & 3,6 & 19,0 & 40,8 & 31,5 & 16,9 & 89,7 \\
\hline Berge artificielle & & & & & & & & \\
\hline Matériaux divers & 0,0 & 2,2 & 0,0 & 2,2 & 0,0 & 10,3 & 0,0 & 10,3 \\
\hline Enrochement & 0,0 & 0,0 & 0,0 & 0,0 & 0,0 & 0,0 & 0,0 & 0,0 \\
\hline Total & 0,0 & 2,2 & 0,0 & 2,2 & 0,0 & 10,3 & 0,0 & 10,3 \\
\hline TOTAL & 8,7 & 8,9 & 3,6 & 21,2 & 40,8 & 42,3 & 16,9 & 100,0 \\
\hline TOTAL (amont/aval) & 58,7 & 57,4 & 19,4 & 135,6 & 42,2 & 42,5 & 15,3 & 100,0 \\
\hline
\end{tabular}


Figure 4a. Sapement à la base d'un talus entraînant progressivement le déchaussement des arbres (rive droite $\mathrm{km} \mathrm{62,4).}$

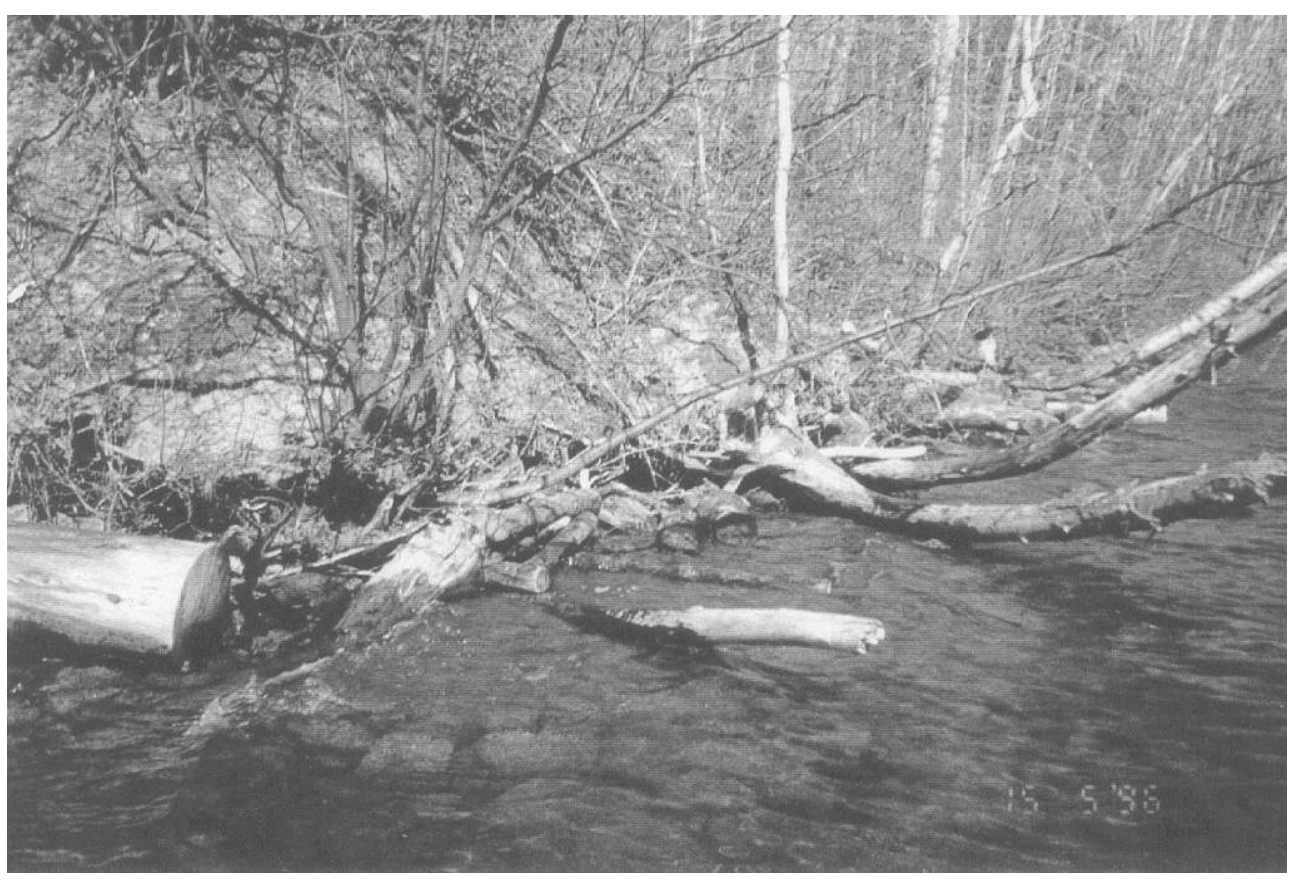

Figure 4b. Éboulements le long des pentes riveraines. Les éboulements entraînent aussi la chute des arbres au pied du talus (rive droite $\mathrm{km} 75,4$ ).




Tableau 5. Longueur des berges en fonction de la hauteur des talus pour les zones constituées de matériaux meubles.

\begin{tabular}{|c|c|c|c|c|c|c|c|c|}
\hline \multicolumn{9}{|c|}{ AMONT } \\
\hline HAUTEUR DU & \multicolumn{3}{|c|}{ LONGUEUR DES BERGES $(\mathrm{km})$} & \multicolumn{5}{|c|}{$\begin{array}{l}\text { POURCENTAGE DE LA LONGUEUR TOTALE } \\
\text {. }\end{array}$} \\
\hline & $\begin{array}{c}\text { Rive } \\
\text { gauche }\end{array}$ & Rive droite & Iles & Total & $\begin{array}{c}\text { Rive } \\
\text { gauche }\end{array}$ & Rive droite & Iles & total \\
\hline $\begin{array}{l}\text { Talus bas }(<2 \\
\mathrm{m})\end{array}$ & 14,7 & 8,4 & 15,6 & 38,7 & 19,8 & 11,2 & 20,8 & 51,8 \\
\hline $\begin{array}{l}\text { Talus } \\
\text { moyennement } \\
\text { élevé }(2-10 \mathrm{~m})\end{array}$ & 6,8 & 10,6 & 0,0 & 17,4 & 9,1 & 14,2 & 0,0 & 23,3 \\
\hline $\begin{array}{l}\text { Talus élevé } \\
(10-20 \mathrm{~m})\end{array}$ & 1,9 & 8,9 & 0,0 & 10,8 & 2,5 & 11,9 & 0,0 & 14,4 \\
\hline Talus très élevé & 2,0 & 5,8 & 0,0 & 7,8 & 2,7 & 7,8 & 0,0 & 10,5 \\
\hline Total & 25,4 & 33,7 & 15,6 & 74,7 & 34,1 & 45,1 & 20,8 & 100,0 \\
\hline \multirow{3}{*}{$\begin{array}{l}\text { HAUTEUR DU } \\
\text { TALUS }\end{array}$} & \multicolumn{8}{|c|}{ AVAL } \\
\hline & \multicolumn{3}{|c|}{ LONGUEUR DES BERGES $(\mathrm{km})$} & \multicolumn{5}{|c|}{ POURCENTAGE DE LA LONGUEUR TOTALE } \\
\hline & $\begin{array}{c}\text { Rive } \\
\text { gauche }\end{array}$ & Rive droite & Iles & Total & $\begin{array}{c}\text { Rive } \\
\text { gauche }\end{array}$ & Rive droite & lles & total \\
\hline $\begin{array}{l}\text { Talus bas }(<2 \\
\mathrm{m})\end{array}$ & 2,5 & 1,1 & 1,1 & 4,7 & 23,8 & 11,5 & 9,5 & 44,8 \\
\hline $\begin{array}{l}\text { Talus } \\
\text { moyennement } \\
\text { élevé }(2-10 \mathrm{~m})\end{array}$ & 3,2 & 2,2 & 0,0 & 5,4 & 30,5 & 20,9 & 0,0 & 51,4 \\
\hline $\begin{array}{l}\text { Talus élevé } \\
(10-20 \mathrm{~m})\end{array}$ & 0,0 & 0,4 & 0,0 & 0,4 & 0,0 & 3,8 & 0,0 & 3,8 \\
\hline Talus très élevé & 0,0 & 0,0 & 0,0 & 0,0 & 0,0 & 0,0 & 0,0 & 0,0 \\
\hline Total & 5,7 & 3,7 & 1,1 & 10,5 & 54,3 & 36,2 & 9,5 & 100,0 \\
\hline $\begin{array}{l}\text { TOTAL } \\
\text { (amont/aval) }\end{array}$ & 31,1 & 37,4 & 16,7 & 85,2 & 44,2 & 40,1 & 15,7 & 100,0 \\
\hline
\end{tabular}

Tableau 6 . Longueur des berges suivant l'inclinaison des pentes pour les zones constituées de matériaux meubles.

\begin{tabular}{|c|c|c|c|c|c|c|c|c|}
\hline \multicolumn{9}{|c|}{ AMONT } \\
\hline $\begin{array}{l}\text { INCLINAISON } \\
\text { DE LA PENTE }\end{array}$ & \multicolumn{3}{|c|}{ LONGUEUR DES BERGES (km) } & \multicolumn{5}{|c|}{ POURCENTAGE DE LA LONGUEUR TOTALE } \\
\hline & $\begin{array}{c}\text { Rive } \\
\text { gauche }\end{array}$ & Rive droite & lles & Total & $\begin{array}{c}\text { Rive } \\
\text { gauche }\end{array}$ & Rive droite & lles & total \\
\hline $\begin{array}{l}\text { Pente faible }(< \\
\left.5^{\circ}\right)\end{array}$ & 4,4 & 0,7 & 10,0 & 15,1 & 5,9 & 0.9 & 13,4 & 20,2 \\
\hline $\begin{array}{l}\text { Pente } \\
\text { moyememement } \\
\text { inclinée }\left(5 \cdot 14^{\circ}\right)\end{array}$ & 2,0 & 3,2 & 0,5 & 5,7 & 2,6 & 4,3 & 0,7 & 7,6 \\
\hline $\begin{array}{l}\text { Pente abrupte } \\
\left(14-25^{\circ}\right)\end{array}$ & 2,3 & 1,0 & 4,6 & 7,9 & 3,1 & 1,3 & 6,2 & 10,6 \\
\hline $\begin{array}{l}\text { Pente très } \\
\text { abrupte }\left(>25^{\circ}\right)\end{array}$ & 16,7 & 28,8 & 0,5 & 46,0 & 22,5 & 38,6 & 0,5 & 61,6 \\
\hline Total & 25,4 & 33,7 & 15,6 & 74,7 & 34,1 & 45,1 & 20,8 & 100,0 \\
\hline \multirow{3}{*}{$\begin{array}{c}\text { HAUTEUR DU } \\
\text { TALUS }\end{array}$} & \multicolumn{8}{|c|}{ AVAL } \\
\hline & \multicolumn{3}{|c|}{ LONGUEUR DES BERGES (km) } & \multicolumn{5}{|c|}{ POURCENTAGE DE LA LONGUEUR TOTALE } \\
\hline & $\begin{array}{c}\text { Rive } \\
\text { gauche }\end{array}$ & Rive droite & lles & Total & $\begin{array}{c}\text { Rive } \\
\text { gauche }\end{array}$ & Rive droite & Iles & total \\
\hline $\begin{array}{l}\text { Pente faible }(< \\
\left.5^{\circ}\right)\end{array}$ & 0,5 & 0,1 & 0,0 & 0,6 & 4,7 & 1,0 & 0,0 & 5,7 \\
\hline $\begin{array}{l}\text { Pente } \\
\text { moyennement } \\
\text { inclinée }\left(5-14^{\circ}\right)\end{array}$ & 1,8 & 1,0 & 1,1 & 3,9 & 17,2 & 9,5 & 9,5 & 36,2 \\
\hline $\begin{array}{l}\text { Pente abrupte } \\
\left(14-25^{\circ}\right)\end{array}$ & 0,1 & 0,1 & 0,0 & 0,2 & 1,0 & 1,0 & 0,0 & 2,0 \\
\hline $\begin{array}{l}\text { Pente très } \\
\text { abrupte }\left(>25^{\circ}\right)\end{array}$ & 3,3 & 2,5 & 0,0 & 5,8 & 31,4 & 24,7 & 0,0 & 51,6 \\
\hline Total & 5,7 & 3,7 & 1,1 & 10,5 & 54,3 & 36,2 & 9,5 & 100,0 \\
\hline $\begin{array}{l}\text { TOTAL } \\
\text { (amont/aval) }\end{array}$ & 31,1 & 37,4 & 16,7 & 85,2 & 44,2 & 40,6 & 15,2 & 100,0 \\
\hline
\end{tabular}


Tableau 7. Longueur et pourcentage des berges en fonction des classes d'érosion.

\begin{tabular}{|c|c|c|c|c|c|c|c|c|}
\hline \multicolumn{9}{|c|}{ AMONT } \\
\hline CLASSES & \multicolumn{3}{|c|}{ LONGUEUR DES BERGES (km) } & \multicolumn{5}{|c|}{ POURCENTAGE DE LA LONGUEUR TOTALE } \\
\hline & $\begin{array}{c}\text { Rive } \\
\text { gauche }\end{array}$ & Rive droite & lles & Total & $\begin{array}{c}\text { Rive } \\
\text { gauche }\end{array}$ & Rive droite & lles & Total \\
\hline Négligeable & 13,5 & 12,8 & 14,4 & 40,7 & 18 & 19 & 19 & 55 \\
\hline Faible & 7,8 & 8,3 & 1,3 & 17,4 & 10 & 2 & 11 & 23 \\
\hline Moyenne & 1,8 & 5,9 & . & 7,7 & 2 & . & 8 & 10 \\
\hline Forte & 2,3 & 6,6 & - & 8,9 & 3 & . & 9 & 12 \\
\hline \multirow[t]{3}{*}{ Total } & 25,4 & 33,6 & 15,7 & 74,7 & 34 & 21 & 45 & 100 \\
\hline & & & & & & & & \\
\hline & \multicolumn{8}{|c|}{ AVAL } \\
\hline HAUTEUR DU & \multicolumn{3}{|c|}{ LONGUEUR DES BERGES (km) } & \multicolumn{5}{|c|}{ POURCENTAGE DE LA LONGUEUR TOTALE } \\
\hline & $\begin{array}{c}\text { Rive } \\
\text { gauche }\end{array}$ & Rive droite & lles & Total & $\begin{array}{c}\text { Rive } \\
\text { gauche }\end{array}$ & Rive droite & Iles & Total \\
\hline Négligeable & 4,9 & 3,1 & 1,0 & 9,0 & 47 & 30 & 10 & 87 \\
\hline Faible & 0,9 & 0,6 & . & 1,4 & 7 & 6 & . & 13 \\
\hline Moyenne & . & - & - & - & . & . & . & . \\
\hline Forte & . & . & . & . & - & . & . & . \\
\hline Total & 5,8 & 3,7 & 1,0 & 10,5 & 54 & 36 & 10 & 100 \\
\hline $\begin{array}{l}\text { Berges actives } \\
\text { (amont/aval) }\end{array}$ & 31,2 & 37,8 & 16,7 & 85,2 & 44 & 41 & 15 & 100 \\
\hline $\begin{array}{l}\text { TOTAL } \\
\text { (amont/aval) }\end{array}$ & 58,7 & 57,4 & 19,4 & 135,6 & 42,2 & 42,5 & 15,3 & 100 \\
\hline
\end{tabular}

\section{Taux de recul des berges}

\section{Taux de recul mesurés à partir de la numérisation des photos aériennes}

La numérisation des photos aériennes (années 1964, 1987 ou 1993) a permis d'évaluer le recul des berges pour trois segments riverains largement affectés par l'érosion riveraine dans le bief amont de la centrale de Grand-Mère. Le taux de recul des trois segments


de recul le plus important, soit entre 15 et 20 mètres pour une période de près de 30 ans (soit de 1964 à 1993). Vient ensuite, le deuxième segment (km 64,0 au km 67,0) avec un recul évalué entre 5 et 15 mètres pour la même période. Enfin le troisième segment $(\mathrm{km}$ 69,6 au km 70,5) montre un recul inférieur aux autres segments (tableau 8), s'expliquant sans doute par une période plus courte d'analyse (de 1964 à 1987). En effet, pour ce dernier site, on peut penser que le taux de recul pourrait être équivalent aux deux autres segments riverains, si la période couverte avait été de la même durée, soit près de trente années. En fonction de ces données, on peut évaluer un taux d'érosion annuel moyen pour ces segments riverains de 0,5 mètres par année environ. Soulignons que ces trois segments de rivière sont les plus affectés par l'érosion pour le secteur étudié. Le recul des berges à ces endroits s'explique essentiellement par deux processus bien identifiés : (1) il y a d'abord le sapement progressif du pied du talus causé par les vagues et les courants ; (2) ensuite, le surcreusement de la berge va entraîner une rupture de pente, causant ainsi un éboulement ou un glissement de terrain. Ce type de phénomène a été observé sur différentes rivières ou réservoirs du Québec dont les berges étaient constituées de haut 
talus argileux ou limono-argileux (Saint-Laurent 1999; Saint-Laurent et Guimont, 1999 ; Saint-Laurent et al., 2001). Les taux de recul obtenus pour les berges constituées de matériaux argileux, en région nordique (portion aval de la Grande Rivière) par exemple, sont de l'ordre de 0,35 et 0,50 m/an (Saint-Laurent et Guimont, 1999), ce qui est comparable à ceux obtenus pour les segments riverains étudiés pour la rivière SaintMaurice (soit 0,50 m/an) dans les zones actives d'érosion.

Tableau 8 . Évaluation du taux de recul des berges obtenus par la numérisation de photographies aériennes.

\begin{tabular}{|c|c|c|c|c|c|c|c|}
\hline $\begin{array}{l}\text { SEGMENTS } \\
\text { RIVERAINS }\end{array}$ & $\begin{array}{l}\text { Classe } \\
\text { d'érosion }\end{array}$ & $\begin{array}{l}\text { Nature } \\
\text { des } \\
\text { matériaux }\end{array}$ & $\begin{array}{l}\text { Pente } \\
\text { des } \\
\text { talus }\end{array}$ & $\begin{array}{l}\text { Hauteur } \\
\text { des } \\
\text { talus } \\
\text { (m) }\end{array}$ & $\begin{array}{l}\text { Recul } \\
\text { des } \\
\text { berges } \\
(\mathrm{m})\end{array}$ & $\begin{array}{l}\text { Taux de } \\
\text { recul } \\
\text { annuel } \\
\text { (m) }\end{array}$ & $\begin{array}{l}\text { Années } \\
\text { étudiées }\end{array}$ \\
\hline $\begin{array}{l}\mathrm{km} 51,8 \text { au } \\
\mathrm{km} 52,8\end{array}$ & Forte & $\begin{array}{l}\text { Argile } \\
\text { silteuse }\end{array}$ & $>25^{\circ}$ & 10 & $15-20$ & $0,5-0,7$ & $\begin{array}{l}1964 . \\
1993\end{array}$ \\
\hline $\begin{array}{l}\mathrm{km} 64,0 \text { au } \\
\mathrm{km} 67,0\end{array}$ & $\begin{array}{l}\text { Forte à } \\
\text { moyenne }\end{array}$ & $\begin{array}{l}\text { Sable et } \\
\text { gravier }\end{array}$ & $>25^{\circ}$ & $2-6$ & $5-15$ & $0,2-0,5$ & $\begin{array}{l}1964- \\
1993\end{array}$ \\
\hline $\begin{array}{l}\mathrm{km} 69,6 \text { au } \\
\mathrm{km} 70,5\end{array}$ & Forte & $\begin{array}{l}\text { Sable } \\
\text { sur argile } \\
\text { silteuse }\end{array}$ & $>25^{\circ}$ & $6-8$ & $2 \mathrm{a}>10$ & $0,08-0,4$ & $\begin{array}{l}1964 . \\
1987\end{array}$ \\
\hline
\end{tabular}

\section{Principaux agents d'érosion riveraine}

\section{Action des vagues}

De façon générale, les vagues générées par les vents dépendent de divers facteurs qui sont: la vitesse du vent, la durée pendant laquelle le vent souffle, la longueur du plan d'eau sur lequel il souffle (fetch) et la profondeur d'eau (Kachugin, 1966 ; Donelan, 1980 ; Thorne, 1982 ; Lawson, 1985 ; Gatto, 1988, 1990 ; Smith, 1991). Ces différents paramètres ont été considérés pour mesurer la force érosive des vagues sur les berges les plus exposées, soit au km 50,5 (rive gauche) et au km 69, 9 (rive droite), lesquelles présentent des signes de forte activité. On notera que les vents de plus forte vélocité proviennent de l'est, du sud-ouest et du sud et ce, pour l'ensemble des récurrences (figure 2a). Les calculs servant à mesurer la force érosive des vagues ont été effectués en utilisant les vents maximaux annuels $(\mathrm{km} / \mathrm{h})$ avec une récurrence de 2 ans. Les données obtenues indiquent que pour certaines directions de vents l'érosion est plus prononcée (tableaux 2a et $2 b$ ). Les berges situées en rive droite $(\mathrm{km} 69,9)$ par exemple, sont soumises à l'érosion des vagues à partir d'une profondeur de 6,0 mètres ou moins (tableau 2a), et ce sont des vents en provenance du nord et de l'est (nord-est, est et sud-est) qui sont les plus effectifs. En

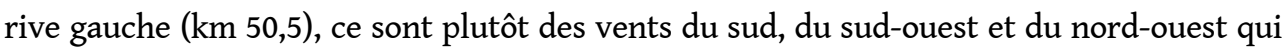
causent l'érosion, et ce à partir d'une profondeur de 2,9 mètres ou moins (tableau 2a). Bien que ce soient les vents en provenance du sud-ouest et du sud-sud-ouest qui sont les plus fréquents (figure $2 b$ ), on remarque que les vents des quadrants de l'est et du nord peuvent générer une érosion importante des berges (tableau 2a). On peut sans doute expliquer ce phénomène au fait que les vents d'est sont généralement associés à des vents de tempête qui génèrent habituellement de fortes vagues. Les berges en rive droite soumises à ces vents de tempête seraient ainsi plus vulnérables à l'érosion (tableau 2a). 
Enfin, les vents d'intensité moyenne n'induisent aucune érosion significative selon les données obtenues (tableau $2 \mathrm{~b}$ ).

Figure 2a. Vent horaire maximal $(\mathrm{km} / \mathrm{h})$ du secteur d'étude.

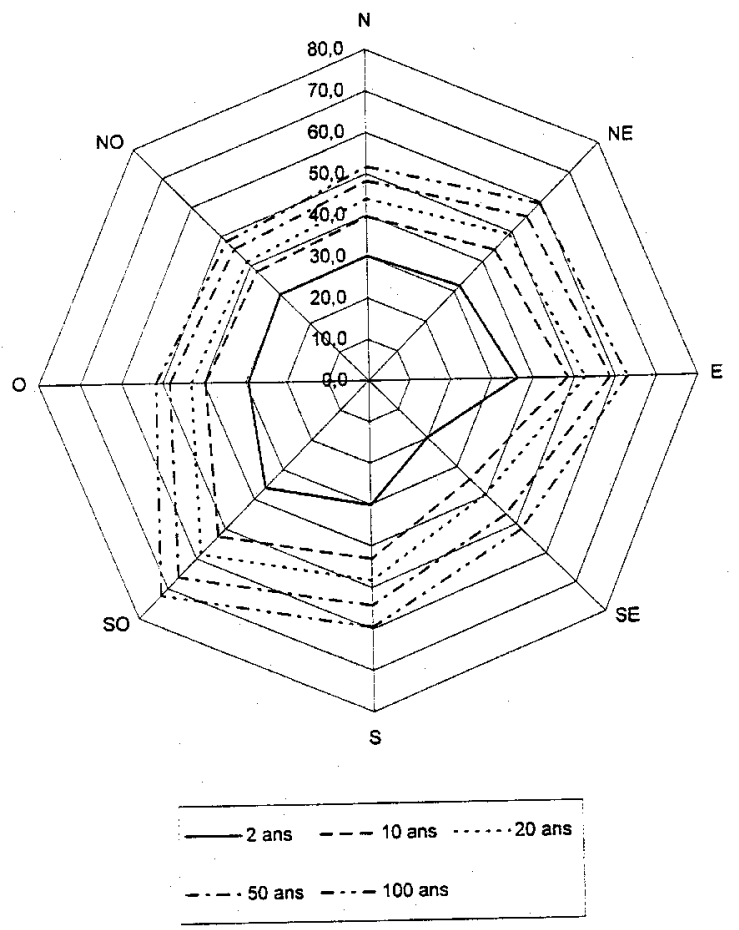

Figure $2 b$. Fréquence des vents (secteur d'étude). 


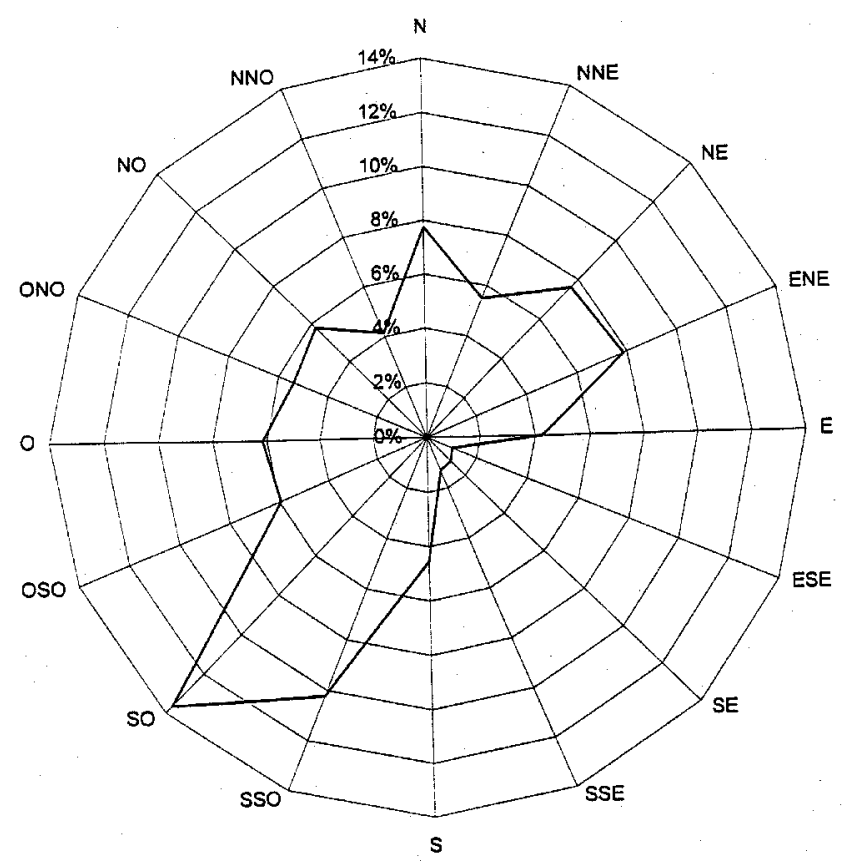

\section{Action des glaces}

L'action des glaces peut avoir un double impact sur les berges; elles peuvent à certaines occasions soit créer une protection ou encore entraîner une érosion des berges (Penner et Sweldo, 1974 ; Lawson, 1985 ; Reid, 1985). En effet, un couvert de glace continu peut avoir pour effet de protéger les berges des courants, mais par contre, un couvert de glace morcelé qui se déplace sous l'effet des vents de tempête ou lors des crues printanières, peut entraîner de l'érosion le long des berges. Sur la rivière Saint-Maurice, l'érosion causée par les glaces se limite principalement à l'impact des glaces flottantes qui viennent saper les berges (Guerrero, 1995 ; Hydro-Québec, 1995). À l'aval de la centrale, l'apparition des glaces de rive se produit vers la fin de novembre. Les glaces se forment à la base des talus des berges puisque le niveau d'eau se situe près de sa limite inférieure durant cette période de l'année (H-Q, 1996c). Lors de la crue le niveau augmente rapidement, soulevant les glaces, ce qui, pendant cette période, peut entraîner l'érosion des berges par arrachement des matériaux pris dans les glaces de rive. Dans le tronçon lotique, entre les rapides des Hêtres et la centrale de Grand-Mère, les glaces se déplacent avec les courants des crues printanières, et ce sont davantage les zones en saillie, comme les pointes ou la partie amont des îles qui se trouvent affectées par les glaces. En amont de la centrale, l'écoulement des eaux se fait plus lentement et le couvert de glace est plus stable (Guerrero, 1995). Lors du dégel, ce couvert de glace fond sur place habituellement, causant ainsi peu d'érosion le long des berges.

\section{Action du batillage}

Outre les vagues, les courants et les glaces, le batillage généré par le passage des bateaux contribue tout aussi à l'érosion des berges. L'érosion provoquée par le passage des 
embarcations motorisées dépend de la taille du bateau, de sa vitesse, de la fréquence des passages et de la distance des embarcations par rapport à la rive. Différentes études réalisées dans le tronçon du fleuve Saint-Laurent (Brebner, 1967; D’Agnalo, 1978; Dauphin, 2000) ont démontré l'effet du batillage sur l'érosion des berges. Auparavant, la présence des estacades de flottage de bois le long des rives de la rivière Saint-Maurice protégeaient en quelque sorte les berges de l'effet du batillage et des vagues, mais avec le démantèlement récent des estacades, soit depuis 1997 et 1998, les berges risquent de devenir plus vulnérables à l'érosion, en particulier si la navigation de plaisance augmente sur la rivière.

\section{Conclusion}

41 L'étude morpho-sédimentologique des berges et des caractéristiques hydrodynamiques de la rivière Saint-Maurice, entre les rapides Manigance et les rapides des Hêtres a permis d'identifier les zones actives d'érosion des berges et de mieux comprendre les processus en cause. Dans ce secteur de la rivière, on évalue à plus de $62 \%$ de berges actives, ce qui représente $85,2 \mathrm{~km}$ de longueur sur un total de $135,6 \mathrm{~km}$. Toutefois, les berges fortement érodées représentent seulement $12 \%$, soit $8,9 \mathrm{~km}$ de longueur dans le bief amont, et sont localisées principalement en rive droite. Ces berges sont principalement constituées de matériaux silto-argileux ou d'argile silteuse avec des pentes généralement élevées et fortement inclinées. En raison de la forte inclinaison des pentes, ces berges sont plus souvent affectées par des éboulements ou des glissements, bien que le sapement demeure l'agent d'érosion le plus important dans l'érosion des berges de tout ce secteur. Les mesures effectuées à partir des photographies aériennes sur les segments riverains les plus érodées, indiquent un taux de recul qui peut atteindre jusqu'à 10 à 15 mètres pour une période de près de 30 ans. Dans le secteur compris entre la centrale et les rapides des Hêtres, le nombre de berges actives est beaucoup plus faible, représentant moins de $10 \%$, soit 10,4 km de longueur de rive. Dans ce secteur, les berges sont surtout constituées en grande partie de roc et de matériaux sableux en pente douce.

L'érosion des berges causée par l'effet des vagues et des glaces se manifeste quelque peu différemment dans les biefs amont et aval. Les berges en aval de la centrale sont davantage affectées par l'action des glaces que celles en amont. Le transport des glaces étant en effet plus important dans cette section de la rivière. En amont, l'action des vagues constitue un agent d'érosion très présent, en particulier dans les zones fortement exposées aux vents dominants. Par ailleurs, l'érosion des berges dans le bief amont risque d'augmenter en raison du batillage générée par les embarcations motorisées. Rappelons que cette section de la rivière a été récemment libérée des estacades (1997-1998) qui jouaient un rôle de protection. On peut donc penser que l'augmentation de la fréquence des bateaux de plaisance, maintenant libres de circuler sur la rivière, entrainera une augmentation du batillage et conséquemment une augmentation de l'érosion riveraine.

\section{Photographies aériennes}

Ministère de l'Énergie et des Ressources du Québec et Hauts Monts Inc. : Q87405, 1 :15 000, 1987, Infrarouge ; Q87406, 1 :15 000, 1987, Infrarouge Q87408, $1: 15$ 000, 1987, Infrarouge ; Q87409, 1 :15 000, 1987, Infrarouge Q87415, 1 :15 000, 1987, Infrarouge ; Q87422, $1: 15$ 000, 1987, Infrarouge Q87425, $1: 15$ 000, 1987, Infrarouge ; Q87427, $1: 15$ 000, 1987, Infrarouge Q87428, $1: 15$ 000, 1987, Infrarouge ; Q87429, 1 :15 000, 1987, Infrarouge 
HMQ93101, $1: 15$ 000, 1993, Noir et blanc ; HMQ93101, $1: 15$ 000, 1993, Noir et blanc HMQ93102, $1: 15$ 000, 1993, Noir et blanc ; HMQ93102, $1: 15$ 000, 1993, Noir et blanc HMQ93104, $1: 15$ 000, 1993, Noir et blanc ; HMQ93104, $1: 15$ 000, 1993, Noir et blanc HMQ93110, $1: 15$ 000, 1993, Noir et blanc ; HMQ93111, $1: 15$ 000, 1993, Noir et blanc HMQ93112, $1: 15$ 000, 1993, Noir et blanc ; HMQ93112, $1: 15$ 000, 1993, Noir et blanc

\section{BIBLIOGRAPHIE}

BREBNER A. (1967), A study of Ship Generated Waves in the St. Lawrence Rive, Department of Civil Engineering, Queens University, Kingston, Ontario, 16 p.

D'AGNOLO F. (1978), Étude d'érosion - Partie du fleuve St-Laurent entre Montréal et Sorel, Garde Côtière canadienne, Ministère des Transports, Canada, 23 p.

DAUPHIN D. (2000), Influence de la navigation commerciale et de la navigation de plaisance sur l'érosion des rives du fleuve Saint-Laurent dans le tronçon Cornwall-Montmagny, Rapport final, ministère des transports du Québec, 103 p. + cartes et annexes.

DONELAN M.A. (1980), Similarity, Theory Applied to the Forecasting of Wave Heights, Periods and Directions, Proceeding of the Canadian Coastal Conference, National Research Council of Canada, pp. 46-61.

ENVIRONNEMENT CANADA (1982), Normales climatiques au Canada, Service de l'Environnement atmosphérique, période 1960-1981, volume 5, Vent, 281 p.

GATTO L.R. (1988), Techniques for measuring reservoir bank erosion, U.S army Corps of Engineers, Cold Regions, Research and Engineering Laboratory, special report, 27 p.

GATTO L.R. (1990), Predicting reservoir bank erosion, a review of methods, Association of Engineering Geologists, United States, $50 \mathrm{p}$.

GROUPE-CONSEIL LASALLE Inc. (1999), Conditions de glace en aval de la centrale et estacade aux rapides des Hêtres, Rapport $\mathrm{n}^{\circ} \mathrm{R} 1354$, préparé pour Hydro-Québec, 17 p. + annexes.

GROUPE HBA EXPERTS-CONSEILS (1993), Centrale Rapide-des-Forges, Étude préliminaire complémentaire, volets géomorphologie et hydrogéologie, Rapport final préparé pour Hydro-Québec, Drummondville, 60 p. + annexes.

GUERRERO A. (1995), Grand-Mère, avant-projet, phase II - Résumé des observations des couverts de glace du Bas Saint-Maurice, Service hydraulique, Hydro-Québec, Rapport n 14320-RA-95-49.

HYDRO-QUEBEC (1993a), Aménagement hydroélectrique Grand-Mère - Relevés des berges du SaintMaurice (érosion et stabilité), Direction aménagement de centrales, Service géologie et structures, rapport préliminaire, $43 \mathrm{p} .+$ annexes et plans.

HYDRO-QUEBEC (1993b), Grand-Mère - Avant-Projet - Phase 1 - Étude du régime des glaces, Direction aménagement de centrales, Service hydraulique, rapport 07442-RA-93/30, 17 p. + annexes.

HYDRO-QUEBEC (1995), Centrale Gand-Mère, avant-projet, phase II - Investigations géotechniques pour ouvrages projetés, Volume 2, prélèvements d'échantillons pour caractérisation environnementale, référence no. 270-018-001, 51 p. + annexes. 
HYDRO-QUEBEC (1996a), Aménagement hydroélectrique Grand-Mère -Étude des berges du réservoir, région Mauricie, secteur des Cascades, Direction ouvrages civils, Service géologie, géotechnique et accès, rapport technique.

HYDRO-QUEBEC (1996b), Avant-projet, centrale Grand-Mère, phase II - Étude sur la stabilité des couverts de glace, rapport $\mathrm{n}^{\circ}$ 14330-RA-96-02.

HYDRO-QUEBEC (1996c), Avant-projet, centrale Grand-Mère, phase II - Études hydrauliques, rapport final.

HYDRO-QUEBEC (1999), Nouvel aménagement hydroélectrique de Grand-Mère, Complément du rapport d'avant-projet, avril, 1999, 201 p. + annexes.

KACHUGIN E.F. (1966), The destructive action of waves on the water-reservoir banks, International Association of Hydrological Sciences, Symposium, Garda, 1, pp. 511-517.

KOMAR P.D. ET MILLER M.C. (1973), « The threshold of sediment movement under oscillatory water waves ", Journal of Sedimentary Petrology, 43, 4, pp. 1101-1110.

LAWSON D.E. (1985), Erosion of Northern Reservoir Shores, an Analysis et Application of Pertinent Literature, US Army Corps of Engineers, Cold Regions Research et Engineering Laboratory, National Technical Information Service (CRREL, Monograph 85-1), 207 p.

PENNER F. ET SWELDO A. (1974), Lake Winnipeg shoreline erosion, sand movement and ice effects study, Water Resources Branches, Departement of Mines, Resources et Environmental Management, Winnipeg, Manitoba, $110 \mathrm{p}$.

REID J.R. (1985), « Bank erosion processes in a cool temperature environment, Orwell Lake, Minnesota ", Geological society of America Bulletin, 96, 6, pp. 781-792.

SAINT-LAURENT D. (1999), « Rives et formes d'érosion fluviale à l'embouchure de la rivière de Rupert, Baie de James, Québec ", The Canadian Geographer/Le Géographe canadien, Canadian Landform Examples, 43, 4, pp. 439-446.

SAINT-LAURENT D. ET GUIMONT P. (1999), « Dynamique fluviale et évolution des berges du cours inférieur des rivières Nottaway, Broadback et de Rupert », Jamésie (Québec), Géographie Physique et Quaternaire, 53, 3, pp. 1-11.

SAINT-LAURENT D., TOUILEB B.N. SAUCET J.-P., WHALEN A., GAGNON B. ET NZAKIMUENA T. (2001), « Effects of simulated water levels management on shore erosion rates, Case study: Baskatong Reservoir, Québec, Canada », Canadian Journal of Civil Engineering, 28, pp. 482-495.

SOCIETE D'ENERGIE DE LA BAIE JAMES (SEBJ) (1975), Rapport du Comité sur la stabilité des berges de la rivière La Grande entre Fort-George et l'aménagement LG-2 (mille 73), Direction Ingénierie, 48 p.

SOCIETE D'INGENIERIE SHAWINIGAN LTEE (1974), Bas Saint-Maurice - Étude d'érosion et de stabilité des berges, Rapports $n^{\circ}$ 54050-00-74 et 54051-00-74, 37 pages + annexes et plans.

SMITH J.M. (1991), Wind-Wave Generation on restricted fetches, Coastal Engineering Research Center, US Army Corps of Engineers, Mai1991.

TECSULT INC. (1996). Nouvelle centrale de Grand-Mère, Érosion des berges, Rapport présenté à la Viceprésidence Environnement, Hydro-Québec, pagination multiple, annexes + cartes.

THORNE C. R. (1982), « Processes and Mechanisms of River Bank Erosion », in HEY R.D., BATHURST J. C. \& THORNE C.R. (eds.), Gravel-bed Rivers, Wiley, Chichester, pp. 227-259.

VINCENT J.-S. (1989), « Le Quaternaire du sud-est du Bouclier canadien », in FULTON R.J (éd.), Le Quaternaire du Canada et du Groenland, Commission géologique du Canada, Ottawa, Canada, pp. 266-295. 


\section{RÉSUMÉS}

Cette étude a été effectuée dans la portion inférieure de la rivière Saint-Maurice qui constitue l'une des grandes rivières du Québec méridional. L'étude examine les processus et phénomènes riverains qui affectent plusieurs berges de cette portion de la rivière. Les facteurs tels que les vagues et les courants, les variations du niveau d'eau et l'impact des glaces sur l'érosion des berges sont considérés. La zone d'étude couvre spécifiquement le tronçon situé entre les rapides Manigance, au nord de la centrale hydroélectrique de Grand-Mère, et les rapides des Hêtres, au sud. En amont de la centrale, les berges sont surtout constituées de silt argileux ou d'argile silteuse. Plus de la moitié des berges sont actives, c'est-à-dire $74,7 \mathrm{~km}$ sur un total de $114,4 \mathrm{~km}$. En aval de la centrale, les berges sont surtout formées de sable et d'un mélange de sable et gravier. Les berges actives forment moins de $10 \%$, soit environ $10,5 \mathrm{~km}$ de longueur. Une évaluation du taux de recul des berges les plus fortement érodées, à partir de la numérisation de photographies aériennes, a fourni une valeur moyenne d'environ $0,5 \mathrm{~m}$ de recul par année. Les zones qui semblent les plus affectées se retrouvent dans des hautes terrasses d'argile.

This study was made in the lower portion of the Saint-Maurice River, one of the important rivers of Southern Québec. This study examines processes and phenomena who affect several banks of this portion of the river. Factors responsible for bank erosion such as waves and currents, water level changes and ice conditions are examined. The study covers specifically the river section between the Manigance rapids, North of the Grand-Mère hydroelectric power plant, and the des Hêtres rapids, in the South. Upstream of the power plant, banks are mostly made of clay or siltyclay more than half of the banks are active, that is $74,7 \mathrm{~km}$ on a total of $114,4 \mathrm{~km}$. Downstream of the power plant, banks are mostly made of sand or a mixture of sand and gravel. The active banks form less than $10 \%$, or about $10,5 \mathrm{~km}$ in length for this sector. The rate of bank recession assessed by the digitalization of aerial photographs is of about 0,5 metres per year for the most eroded banks. The most affected zones are located in the high terraces of clay.

\section{INDEX}

Mots-clés : érosion riveraine et recul des berges, calcul des vagues érosives, réservoir hydroélectrique, rivière Saint-Maurice, Québec, Canada

Keywords : bank erosion and bank recession, calculation of waves erosion, hydroelectric power plant, Saint-Maurice River

\section{AUTEURS}

\section{DIANE SAINT-LAURENT}

Université du Québec à Trois-Rivières, Section géographie, diane_saint-laurent@uqtr.ca

\section{MICHEL BÉRUBÉ}

Hydro-Québec, Direction Environnement et Services techniques

\section{ISABELLE THÉRIAULT}

Hydro-Québec, Stratégies et plans de production 


\section{MICHEL LEMIEUX}

Tecsult Inc. Section Environnement Québec 